\title{
Short Communication: Bird species in the cement factory complex of Tarjun, South Kalimantan, Indonesia
}

\author{
MAULANA KHALID RIEFANI ${ }^{1, \vartheta}$, MOCHAMAD ARIEF SOENDJOTO ${ }^{2}$, ANDY M. MUNIR \\ ${ }^{1}$ Faculty of Teaching and Educational Sciences, Universitas Lambung Mangkurat. Jl. Hasan Basry, Kayutangi, Banjarmasin 70123, South Kalimantan, \\ Indonesia." email: maulanakriefani@ulm.ac.id \\ ${ }^{2}$ Forestry Faculty, Universitas Lambung Mangkurat. Jl. Ahmad Yani Km 36, Banjarbaru 70714, South Kalimantan, Indonesia \\ ${ }^{3}$ PT Indocement Tunggal Prakarsa Tbk., Tarjun, Kotabaru, Indonesia
}

Manuscript received: 29 September 2018. Revision accepted: 21 December 2018.

\begin{abstract}
Riefani MK, Soendjoto MA, Munir AM. 2019. Bird species in the cement factory complex of Tarjun, South Kalimantan, Indonesia. Biodiversitas 20: 218-225. The factory complex of PT Indocement Tunggal Prakarsa Tbk., Tarjun, South Kalimantan, Indonesia consists of cement manufacturing plants and employee settlements. Supporting facilities for the two main areas are open spaces in the form of waters (lakes, small rivers), grasslands (golf courses, grassy fields with several woody individuals growing on it), and forests (mangrove forests, secondary lowland forests, including shrubs). However, the data related to bird species that live in complex are not available yet. The purpose of this study was to inventory bird species in the ITP complex and to analyze qualitatively the presence of birds. These data will be used as a baseline for the development of the complex, comparative data for birds in the operational area of mining located $27 \mathrm{~km}$ outside the complex, and the completeness of the requirements for submitting a company performance rating (Proper). Birds were inventoried four periods between 2015-2016 at 06:00 - 08:00 and 16:30 - 18:00. The period here was treated as repetition. Observers equipped with binoculars and telephoto cameras walked around through footpaths and roads exploring the whole complex. In water and grassland areas, birds recorded can be at an observation distance of more than $50 \mathrm{~m}$, whereas in forest areas, birds recorded were at a maximum distance of $50 \mathrm{~m}$. A total of eighty-seven species and 41 families of birds were recorded. Of them, eighteen species and 8 families are waterbirds. The most recorded birds were classified as residents. All of the birds were recorded or observed easily every day or almost every day, at least at the research sites. Others ( 8 species) wee categorized as migratory birds. Fourteen species are protected by the Regulation of the Minister of Environment and Forestry, the Republic of Indonesia No. P.92/MENLHK/SETJEN/KUM.1/8/2018, 30 August 2018.
\end{abstract}

Keywords: Birds inventory, PT Indocement Tunggal Prakarsa, open space, settlement, South Kalimantan.

\section{INTRODUCTION}

PT Indocement Tunggal Prakarsa Tbk. (ITP) is the second largest cement producer in Indonesia (2018b) and operates three cement-producing factories (Heidelberg Cement Group 2018). One factory (with 10 plants) is located at Citeureup, Bogor, West Java Province, 1 factory (with 2 plants) at Palimanan, Cirebon, West Java Province, and the other factory (1 plants) at Tarjun Village, Kelumpang Hilir District, Kotabaru Regency South Kalimantan Province.

The Tarjun Factory Complex lies on the shore of Laut Strait, a narrow sea that extends from north to south and separates Laut Island in a total of $\pm 1.873,36 \mathrm{~km}^{2}$ (east) from Borneo which covers a total of $743.330 \mathrm{~km}^{2}$ (west). Borneo is the third-largest island in the world and controlled by Indonesia, Malaysia, and Brunei Darussalam. The ITP factory location is strategic. From a plant with a production capacity of 2.6 million tons per year (ITP 2018c), cement both in packaging and in bulk form can be loaded directly to the ship to be transported to distributors and retailers in Java, Sumatra, Kalimantan, Sulawesi and other islands in Indonesia.
The ITP complex covering an area of about 586 ha (ITP 2016, 2017) consists of two main areas, namely cement manufacturing plants and employee settlements. Supporting facilities for two main areas are open spaces in the form of waters (lakes, ponds, creeks), grassland areas (golf courses, soccer fields, grass fields with some woody individuals growing on them), forests (mangroves, dry-land forests, including shrubs and nursery gardens). The function of these supporting facilities certainly makes this area safe and ecologically safe, especially for factory employees and buyers from the surrounding areas (in South Kalimantan and East Kalimantan Province) who come directly to drive cement transport vehicles (trucks), and anyone who gets permission to enter the ITP complex. Employees can work according to or even exceed their performance targets and find their nuclear families healthy and not anxious as long as they are present or even absent from their daily lives. Buyers can also use their free time in stress-free conditions while waiting to get calls from the factory authority or waiting for their turn to load cement into their transport vehicles.

On the other hand, the supporting facilities become a safe and comfortable place for plants and animals. The birds currently discussed can do all kinds of behavior 
without fear of being hunted or consumed by people. Birds can play, search food, and breed to keep their population preserved or even increase. Moreover, the company has committed itself to conserving biodiversity and natural resources whose strategies include preventing extinction of endemic birds at the plant site and planting trees to develop bird habitats (ITP 2015).

Unfortunately, data related to bird species that live in the Tarjun Factory Complex are not sufficient. We did not get environmental documents (such as Environmental Impact Analysis) or official reports containing fauna data (especially in this case, birds) in the Tarjun Factory Complex. Data about birds were actually obtained from two other factory complexes. In the Citeureup Factory Complex 41 species of birds were recorded in 2015 (ITP 2016) and then 83 species in 2016 (ITP 2017) and also in 2017 (ITP 2018d). One of them was Javan Kingfisher (Halcyon cyanoventris), endemic species on the Java Island (ITP 2018d). In the Palimanan Factory Complex 66 species of birds were found in 2016 (ITP 2017) and also in 2017 (ITP 2018d). One of them was Java Sparrow (Padda oryzivora), an endemic species of Java Island and vulnerable based on the International Union for the Conservation of Nature and Natural Resources (IUCN) (ITP 2018d).

The purpose of this study was to inventory bird species in the Tarjun Factory Complex and to qualitatively analyze the factors that caused the existence of birds. Data obtained from this bird inventory activity has multiple benefits. The data will be used as a baseline for the continuous development of the Tarjun Factory Complex, comparative data with bird data that are presented or found in the operational area of cement raw material mining located 27 $\mathrm{km}$ outside the complex, and the completeness of important requirements submitted for the assessment program for company performance (Program Penilaian Peringkat Kinerja Perusahaan, PROPER). The annual assessment program is launched by the Ministry of Environment and Forestry, the Republic of Indonesia to assess the company's compliance with environmental management (related to water management, air management, and management of waste from hazardous and toxic materials), development of community welfare, and clean production. Companies involved in PROPER are those whose industries have an important impact on the environment and their companies care about reputation.

\section{MATERIALS AND METHODS}

Through the opportunistic observation method, birds found at 06:00 - 08:00 and 16:30 - 18:00 in the ITP factory complex of Tarjun in the four periods of 2015-2016 were recorded. This observation hour was actually the free time before and after we observed diurnal birds in the quarry area of cement raw-materials which is $27 \mathrm{~km}$ from the factory complex. The periods, namely P1 (8 - 15 April 2015), P2 (29 July - 4 August 2015), P3 (5 - 12 November
2015), and P4 (28 February - 8 March 2016) were treated as repetitions, even though observation routes in each period were not exactly the same. Habitat types were not differentiated, because we classified the entire factory complex as a modified habitat that was relatively highly influenced by human activity.

Combined transect and concentration count methods were used to be able to explore birds throughout the Tarjun Factory Complex (Figure 1). With the first method, observers recorded birds through paths (in forest areas, areas of tree vegetation, areas where people rarely pass) or paved / rocky roads (infrastructure for general use in the complex). Occasionally the observers stopped for 30 minutes. With the concentration count method, observers not only recorded, but also photographed birds that were within the range of sight. In waters and grassland areas, birds that were recorded can be at an observation distance of more than $50 \mathrm{~m}$, whereas in forest areas, birds recorded at a maximum distance of $50 \mathrm{~m}$. Bird species whose morphology or sounds were known directly by observers were recorded in the databook. However, birds whose morphology and sound were unknown or doubtful were further observed with the help of binoculars or cameras equipped with telelens $(80 \times 400)$ or $(70 \times 300)$. The field guides used to identify birds were MacKinnon et al. (2010) and Soendjoto et al. (2015b). The comparison field guides were Smythies (1981) and Strange (2002). Then the taxonomic names are referred to IUCN (2018).

The data is tabulated in a 14 column table. A column after the serial number column (first column) contains the family and scientific names of the bird species. Both the family name and the species name in this column are arranged in alphabetical A-Z.

Furthermore, birds are classified (i) whether they are waterbirds, (ii) according to their occupancy status, and (iii) according to their protection status. Birds are classified as waterbirds if their morphology refers to the criteria of waterbirds, such as both long feet and long beak (for example Anhingidae, Ardeidae, Charadriidae, Ciconiidae, Rallidae, and Scolopacidae) or have membranes between their fingers (for example Anatidae and Laridae). The bird family taxa (32 families) included in waterbirds are listed by Wetlands International (2012). Bird occupancy status consists of two groups, namely resident and migrant. Bird species is a resident if this bird is recorded or observed easily every day or almost every day, at least in the ITP complex and its surroundings. Migratory bird status refers to MacKinnon et al. (2010). The status of bird protection is determined according to Regulation of the Minister of Environment and Forestry, the Republic of Indonesia No. P.92/MENLHK/SETJEN/KUM.1/8/2018, 30 August 2018, concerning Amendment to Regulation of the Minister of Environment and Forestry No. P.20/MENLHK/SETJEN/KUM.1/6/2018 concerning the Protected Species of Plants and Animals. This is a substitute for Government Regulation No. 7, 27 January 1999 concerning Preservation of Species of Plants and Animals. 


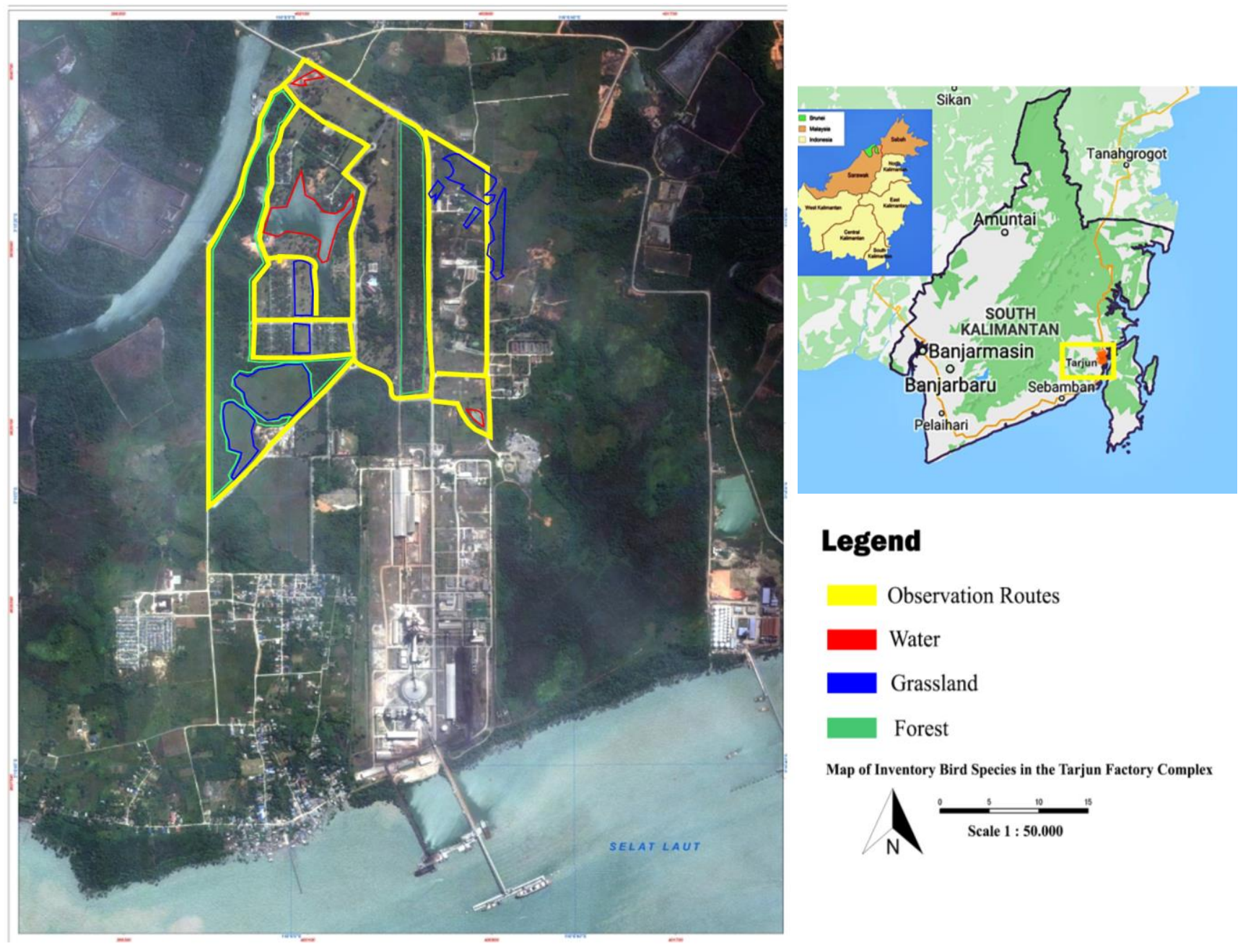

Figure 1. Geographical position of PT. Indocement Tunggal Prakarsa Tbk., Tarjun

\section{RESULTS AND DISCUSSION}

A total of 87 species belongs to 41 families of birds were recorded (Table 1). Of these species, 14 are protected birds and eighteen species ( 8 families) are waterbirds. Most birds are residents (79 of 87 bird species) and others are categorized as migrants. Five of the 8 species of migratory birds are waterbirds, two species are predators, and the rest is Java Sparrow, species specifically in this study are grouped in migrants. Java Sparrow is an endemic species of Java, Bali, and Kangean which was then widely introduced from Southeast Asia to Australia (Smythies 1981; MacKinnon et al. 2010).

These waterbirds can be recorded in the waters of the complex. One of them is Lake Tiro (the acronym of Tiga Roda, Three Wheels in English), which is about 5 ha in size. The widest lake in the factory complex is formed after the flow of water in the river which empties into the Terusan River is dammed. The edge of the lake bordering the settlement, in general, is relatively shallow. Lakeforming mud appears, especially if the volume of lake water decreases or subsidies. In muddy locations that are not overgrown with grass or overgrown with grass, found waterbirds that have long legs and neck and large body sizes such as Great Egret, and Little Egret. In addition, there are also feet and long necks, but small birds, like the Common Sandpiper. At the edge of the lake overgrown with Poaceae (grass family) and Cyperaceae (sedge family) can be found Javan Pond-heron, Striated Heron, Whitebreasted Waterhen, Common Moorhen, and Wood Sandpiper. On cables stretching over the surface of the lake or at the end of wooden pillars that stick to the bottom of the lake, birds like Little Tern and Common Tern often sit. At the edge of the lake near the dam wall which limits lake water with relatively deep rivers, some waterbirds are found swimming. Birds include Oriental Darter, Wandering Whistling-duck, and Sunda Teal. 
Table 1. Bird species recorded in the factory complex of PT Indocement Tunggal Prakarsa Tbk., Tarjun

\begin{tabular}{|c|c|c|c|c|c|c|c|c|c|c|c|}
\hline Family and scientific name & Indonesian name & Common name & $\begin{array}{l}\text { Obs. } \\
\text { P1 P }\end{array}$ & $\begin{array}{l}. \text { peri } \\
22 \mathrm{P3}\end{array}$ & $\begin{array}{ll}\text { iod I } \\
\text { P4 s }\end{array}$ & $\begin{array}{l}\text { IUCN } \\
\text { status }\end{array}$ & $\begin{array}{l}\text { Waterb } \\
\text { Fam. }\end{array}$ & $\begin{array}{l}\text { birds } \\
\text { Sp. }\end{array}$ & Prot. & $\begin{array}{l}\text { Occup } \\
\text { Res. }\end{array}$ & $\begin{array}{l}\text { pancy } \\
\text { Mig. }\end{array}$ \\
\hline \multicolumn{12}{|c|}{ 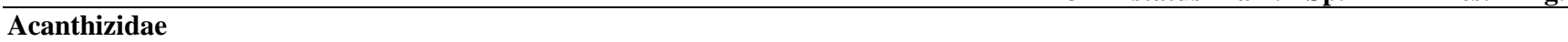 } \\
\hline Gerygone sulphurea & Remetuk laut & Golden-bellied Gerygone & $\bullet$ & $\bullet$ & $\bullet \quad 1$ & LC-d & - & - & - & $\bullet$ & - \\
\hline \multicolumn{12}{|l|}{ Accipitridae } \\
\hline Haliaeetus leucogaster & Elang-laut perut-putih & White-bellied Fish-eagle & - & - & - 1 & LC-d & - & - & - & - & - \\
\hline Haliastur indus & Elang bondol & Brahminy Kite & $\bullet$ & & - 1 & LC-d & - & - & $\bullet$ & $\bullet$ & - \\
\hline \multicolumn{12}{|l|}{ Aegithinidae } \\
\hline Aegithina tiphia & Cipoh kacat & Common Iora & - & - & - $\mathrm{l}$ & LC-u & - & - & - & - & - \\
\hline Aegithina viridissima & Cipoh jantung & Green Iora & $\bullet$ & • & - 1 & NT-d & - & - & - & $\bullet$ & - \\
\hline \multicolumn{12}{|l|}{ Alcedinidae } \\
\hline Alcedo meninting & Raja-udang meninting & Blue-eared Kingfisher & - & - & - 1 & LC-d & - & - & - & $\bullet$ & - \\
\hline Todiramphus chloris & Cekakak sungai & Collared Kingfisher & $\bullet$ & & - I & LC-d & - & - & - & $\bullet$ & - \\
\hline Todiramphus sanctus & Cekakak suci & Sacred Kingfisher & $\bullet$ & & & LC-i & - & - & - & $\bullet$ & - \\
\hline Pelargopsis capensis & Pekaka emas & Stork-billed Kingfisher & $\bullet$ & $\bullet$ & - $\mathrm{I}$ & LC-d & - & - & - & $\bullet$ & - \\
\hline \multicolumn{12}{|l|}{ Anatidae } \\
\hline Anas gibberifrons & Itik benjut & Sunda Teal & - & $\bullet$ & & NT-s & $\bullet$ & $\bullet$ & - & $\bullet$ & - \\
\hline Dendrocygna arcuata & Belibis kembang & Wandering Whistling-duck & - & - & - 1 & LC-d & - & - & - & - & - \\
\hline \multicolumn{12}{|l|}{ Anhingidae } \\
\hline Anhinga melanogaster & Pecuk-ular asia & Oriental Darter & - & - & $\bullet 1$ & NT-d & $\bullet$ & $\bullet$ & $\bullet$ & $\bullet$ & - \\
\hline \multicolumn{12}{|l|}{ Ardeidae } \\
\hline Ardeola speciosa & Blekok sawah & Javan Pond-heron & - & - & - 1 & LC-u & - & - & - & - & - \\
\hline Butorides striata & Kokokan laut & Striated Heron & $\bullet$ & $\bullet$ & - $\mathrm{I}$ & LC-d & - & $\bullet$ & - & $\bullet$ & - \\
\hline Ardea alba & Kuntul besar & Great Egret & - & $\bullet$ & - 1 & LC-u & - & $\bullet$ & - & $\bullet$ & - \\
\hline Egretta garzetta & Kuntul kecil & Little Egret & $\bullet$ & $\bullet$ & & LC-i & - & $\bullet$ & - & $\bullet$ & - \\
\hline Egretta sacra & Kuntul karang & Pacific Reef-egret & - & - & & LC-s & - & $\bullet$ & - & $\bullet$ & - \\
\hline Ixobrychus cinnamomeus & Bambangan merah & Cinnamon Bittern & - & - & & LC-s & - & - & - & - & - \\
\hline Ixobrychus sinensis & Bambangan kuning & Yellow Bittern & - & - & & LC-u & - & - & - & - & - \\
\hline \multicolumn{12}{|l|}{ Artamidae } \\
\hline Artamus leucoryn & Kekep babi & $\begin{array}{l}\text { White-breasted } \\
\text { Woodswallow }\end{array}$ & $\bullet$ & & - 1 & LC-s & - & - & - & $\bullet$ & - \\
\hline \multicolumn{12}{|l|}{ Bucerotidae } \\
\hline Anthracoceros albirostris & Kangkareng perut putih & Oriented Pied Hornbill & - & $\bullet$ & -1 & LC-s & - & - & $\bullet$ & $\bullet$ & - \\
\hline \multicolumn{12}{|l|}{ Campephagidae } \\
\hline Lalage nigra & Kapasan kemiri & Pied Triller & $\bullet$ & $\bullet$ & - 1 & LC-s & - & - & - & $\bullet$ & - \\
\hline Pericrocotus flammeus & Sepah hutan & Scarlet Minivet & - & $\bullet$ & - 1 & LC-s & - & - & - & $\bullet$ & - \\
\hline \multicolumn{12}{|l|}{ Caprimulgidae } \\
\hline Caprimulgus affinis & Cabak kota & Savannah Nightjar & - & $\bullet$ & $\bullet$ & LC-s & - & - & - & $\bullet$ & - \\
\hline \multicolumn{12}{|l|}{ Charadriidae } \\
\hline Charadrius dubius & Cerek-kalung kecil & Little Ringed Plover & - & $\bullet$ & $\bullet$ & LC-s & $\bullet$ & $\bullet$ & - & - & $\bullet$ \\
\hline \multicolumn{12}{|l|}{ Ciconiidae } \\
\hline Leptoptilos javanicus & Bangau tongtong & Lesser Adjutant & $\bullet$ & $\bullet$ & $\bullet$ & Vu-d & $\bullet$ & $\bullet$ & $\bullet$ & $\bullet$ & - \\
\hline \multicolumn{12}{|l|}{ Cisticolidae } \\
\hline Orthotomus ruficeps & Cinenen kelabu & Ashy Tailorbird & - & - & & LC-s & - & - & - & - & - \\
\hline Orthotomus sericeus & Cinenen merah & Rufous-tailed Tailorbird & $\bullet$ & & $\bullet$ & LC-s & - & - & - & - & - \\
\hline Prinia flaviventris & Prenjak rawa & Yellow-bellied Prinia & $\bullet$ & $\bullet$ & - $\mathrm{I}$ & LC-d & - & - & - & $\bullet$ & - \\
\hline Columbidae & & & & & & & & & & & \\
\hline Geopelia striata & Perkutut & Zebra Dove & $\bullet$ & $\bullet$ & - 1 & LC-s & - & - & - & $\bullet$ & - \\
\hline Spilopelia chinensis & Tekukur & Eastern Spotted Dove & $\bullet$ & $\bullet$ & $\bullet$ & LC-i & - & - & - & $\bullet$ & - \\
\hline Treron vernans & Punai gading & Pink-necked Green-pigeon & - & $\bullet$ & $\bullet$ & LC-s & - & - & - & $\bullet$ & - \\
\hline Corvidae & & & & & & & & & & & \\
\hline Corvus macrorhynchos & Gagak kampung & Large-billed Crow & - & - & -1 & LC-s & - & - & - & $\bullet$ & - \\
\hline Cuculidae & & & & & & & & & & & \\
\hline Cacomantis merulinus & Wiwik kelabu & Plantive Cuckoo & - & $\bullet$ & - 1 & LC-s & - & - & - & $\bullet$ & - \\
\hline Centropus bengalensis & Bubut kecil & Lesser Coucal & - & - & $\bullet$ & LC-i & - & - & - & $\bullet$ & - \\
\hline Centropus sinensis & Bubut besar & Greater Coucal & - & $\bullet$ & - 1 & LC-s & - & - & - & $\bullet$ & - \\
\hline Rhinortha chlorophaea & Kadalan selaya & Raffles's Malkoha & - & $\bullet$ & $\bullet$ & LC-s & - & - & - & $\bullet$ & - \\
\hline $\begin{array}{l}\text { Phaenicophaeus } \\
\text { curvirostris }\end{array}$ & Kadalan birah & $\begin{array}{l}\text { Chestnut-breasted } \\
\text { Malkoha }\end{array}$ & - & $\bullet$ & -1 & LC-s & - & - & - & $\bullet$ & - \\
\hline Dicaeidae & & & & & & & & & & & \\
\hline Dicaeum cruentatum & Cabai merah & $\begin{array}{l}\text { Scarlet-backed } \\
\text { Flowerpecker }\end{array}$ & - & - & - & LC-s & - & - & - & $\bullet$ & - \\
\hline Dicaeum trochileum & Cabai jawa & $\begin{array}{l}\text { Scarlet-headed } \\
\text { Flowerpecker }\end{array}$ & $\bullet$ & $\bullet$ & - 1 & LC-s & - & - & - & $\bullet$ & - \\
\hline
\end{tabular}




\begin{tabular}{|c|c|c|c|c|c|c|c|c|c|c|c|}
\hline Dicaeum trigonostigma & Cabai bunga api & $\begin{array}{l}\text { Orange-bellied } \\
\text { Flowerpecker }\end{array}$ & - & $\bullet$ & - & - LC-s & - & - & - & $\bullet$ & - \\
\hline \multicolumn{12}{|l|}{ Estrildidae } \\
\hline Lonchura fuscans & Bondol kalimantan & Dusky Munia & - & - & • & - LC-s & - & - & - & - & - \\
\hline Lonchura malacca & Bondol rawa & Black-headed Munia & - & - & - & - LC-s & - & - & - & - & - \\
\hline Lonchura oryzivora & Gelatik jawa & Java Sparrow & - & - & - & - $\mathrm{Vu}-\mathrm{d}$ & - & - & - & - & - \\
\hline Lonchura punctulata & Bondol peking & Scaly-breasted Munia & $\bullet$ & $\bullet$ & $\bullet$ & - LC-s & - & - & - & $\bullet$ & - \\
\hline \multicolumn{12}{|l|}{ Falconidae } \\
\hline Falco moluccensis & Alap-alap sapi & Spotted Kestrel & - & - & - & - $\mathrm{LC}-\mathrm{i}$ & - & - & - & - & - \\
\hline \multicolumn{12}{|l|}{ Hemiprocnidae } \\
\hline \multicolumn{12}{|l|}{ Hirundinidae } \\
\hline $\begin{array}{l}\text { Hirundo tahitica } \\
\text { Laniidae }\end{array}$ & \multicolumn{5}{|c|}{ Laniidae } & - $\quad \mathrm{LC}-\mathrm{u}$ & - & - & - & $\bullet$ & - \\
\hline $\begin{array}{l}\text { Lanius schach } \\
\text { Laridae }\end{array}$ & Bentet kelabu & Long-tailed Shrike & $\bullet$ & $\bullet$ & $\bullet$ & - $\mathrm{LC}-\mathrm{u}$ & - & - & - & $\bullet$ & - \\
\hline Sterna hirundo & Dara-laut biasa & Common Tern & - & - & $\bullet$ & - $\mathrm{LC}-\mathrm{u}$ & $\bullet$ & $\bullet$ & $\bullet$ & - & $\bullet$ \\
\hline Sternula albifrons & Dara-laut kecil & Little Tern & - & - & $\bullet$ & - LC-d & - & $\bullet$ & $\bullet$ & - & $\bullet$ \\
\hline \multicolumn{12}{|l|}{ Megalaimidae } \\
\hline Psilopogon rafflesii & Takur tutut & Red-crowned Barbet & - & - & - & - NT-d & - & - & - & - & - \\
\hline Caloramphus fuliginosus & Takur ampis & Brown Barbet & - & - & $\bullet$ & - LC-d & - & - & - & $\bullet$ & - \\
\hline \multicolumn{12}{|l|}{ Meropidae } \\
\hline Merops philippinus & Kirik-kirik laut & Blue-tailed Bee-eater & - & - & $\bullet$ & - LC-s & - & - & - & - & - \\
\hline \multicolumn{12}{|l|}{ Motacillidae } \\
\hline Motacilla flava & Kicuit kerbau & Western Yellow Wagtail & - & - & - & - LC-d & - & - & - & - & - \\
\hline Anthus novaeseelandiae & Apung tanah & Common Pipit & - & - & $\bullet$ & - LC-s & - & - & - & $\bullet$ & - \\
\hline \multicolumn{12}{|l|}{ Muscicapidae } \\
\hline Cyornis rufigastra & Sikatan bakau & Mangrove Blue-flycatcher & - & - & - & - LC-d & - & - & - & - & - \\
\hline Muscicapa dauurica & Sikatan bubik & Asian Brown Flycacther & - & - & $\bullet$ & - LC-s & - & - & - & $\bullet$ & - \\
\hline \multicolumn{12}{|l|}{ Nectariniidae } \\
\hline Aethopyga siparaja & Burung-madu sepah raja & Crimson Sunbird & - & - & - & - LC-s & - & - & - & - & - \\
\hline Anthreptes malacensis & Burung-madu kelapa & Plain-throated Sunbird & $\bullet$ & $\bullet$ & $\bullet$ & - LC-s & - & - & - & $\bullet$ & - \\
\hline Anthreptes simplex & Burung-madu polos & Plain Sunbird & - & - & $\bullet$ & - LC-d & - & - & - & $\bullet$ & - \\
\hline Arachnothera longirostra & Pijantung kecil & Little Spiderhunter & - & - & - & - LC-s & - & - & - & $\bullet$ & - \\
\hline Chalcoparia singalensis & Burung-madu belukar & Ruby-cheeked Sunbird & - & - & - & - LC-s & - & - & - & $\bullet$ & - \\
\hline Cinnyris jugularis & Burung-madu sriganti & Olive-backed Sunbird & $\bullet$ & $\bullet$ & • & - LC-s & - & - & - & $\bullet$ & - \\
\hline Leptocoma calcostetha & Burung-madu bakau & Copper-throated Sunbird & $\bullet$ & $\bullet$ & $\bullet$ & - LC-s & - & - & - & $\bullet$ & - \\
\hline \multicolumn{12}{|l|}{ Pandionidae } \\
\hline Pandion haliaetus & Elang tiram & Osprey & - & - & - & - $\mathrm{LC}-\mathrm{i}$ & - & - & - & - & - \\
\hline Paridae & & & & & & & & & & & \\
\hline Parus major & Gelatik batu kelabu & Great Tit & - & - & $\bullet$ & - LC-i & - & - & - & $\bullet$ & - \\
\hline Passeridae & & & & & & & & & & & \\
\hline Passer montanus & Burung gereja & Tree Sparrow & - & - & - & - LC-d & - & - & - & - & - \\
\hline Picidae & & & & & & & & & & & \\
\hline Dinopium javanense & Pelatuk besi & Common Goldenback & $\bullet$ & $\bullet$ & $\bullet$ & - LC-d & - & - & - & - & - \\
\hline Meiglyptes tukki & Caladi badok & Buff-necked Woodpecker & - & - & $\bullet$ & - NT-d & - & - & - & $\bullet$ & - \\
\hline Picoides moluccensis & Caladi tilik & Sunda Woodpecker & $\bullet$ & $\bullet$ & $\bullet$ & - $\mathrm{LC}-\mathrm{i}$ & - & - & - & $\bullet$ & - \\
\hline Chrysophlegma miniaceum & Pelatuk merah & Banded Woodpecker & - & - & • & - LC-s & - & - & - & - & - \\
\hline Psittacidae & & & & & & & & & & & \\
\hline Loriculus galgulus & Serindit melayu & $\begin{array}{l}\text { Blue-crowned Hanging- } \\
\text { Parrot }\end{array}$ & - & - & - & - LC-s & - & - & - & - & - \\
\hline Psittacula longicauda & Betet ekor-panjang & Long-tailed Parakeet & $\bullet$ & $\bullet$ & $\bullet$ & - NT-d & - & - & - & $\bullet$ & - \\
\hline Pycnonotidae & & & & & & & & & & & \\
\hline Pycnonotus aurigaster & Cucak kutilang & Sooty-headed Bulbul & $\bullet$ & $\bullet$ & $\bullet$ & - LC-d & - & - & - & $\bullet$ & - \\
\hline Pycnonotus brunneus & Merbah mata-merah & Red-eyed Bulbul & - & - & - & - LC-d & - & - & - & $\bullet$ & - \\
\hline Pycnonotus goiavier & Merbah cerucuk & Yellow-vented Bulbul & $\bullet$ & $\bullet$ & $\bullet$ & - LC-i & - & - & - & $\bullet$ & - \\
\hline Pycnonotus plumosus & Merbah belukar & Olive-winged Bulbul & $\bullet$ & $\bullet$ & $\bullet$ & - LC-s & - & - & - & $\bullet$ & - \\
\hline Rallidae & & & & & & & & & & & \\
\hline Amaurornis phoenicurus & Kareo padi & White-breasted Waterhen & $\bullet$ & $\bullet$ & $\bullet$ & - $\mathrm{LC}-\mathrm{u}$ & - & $\bullet$ & - & $\bullet$ & - \\
\hline Gallinula chloropus & Mandar batu & Common Moorhen & $\bullet$ & $\bullet$ & - & - LC-s & $\bullet$ & $\bullet$ & - & $\bullet$ & - \\
\hline Rhipiduridae & & & & & & & & & & & \\
\hline Rhipidura javanica & Kipasan belang & Pied Fantail & $\bullet$ & $\bullet$ & $\bullet$ & - LC-s & - & - & - & $\bullet$ & - \\
\hline Scolopacidae & & & & & & & & & & & \\
\hline Actitis hypoleucos & Trinil pantai & Common Sandpiper & - & 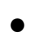 & 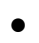 & - LC-d & - & - & - & - & - \\
\hline Tringa glareola & Trinil semak & Wood Sandpiper & - & $\bullet$ & $\bullet$ & - LC-s & $\bullet$ & $\bullet$ & - & - & $\bullet$ \\
\hline Sturnidae & & & & & & & & & & & \\
\hline Acridotheres javanicus & Kerak kerbau & Javan Myna & - & - & - & - Vu-d & - & - & - & - & - \\
\hline
\end{tabular}




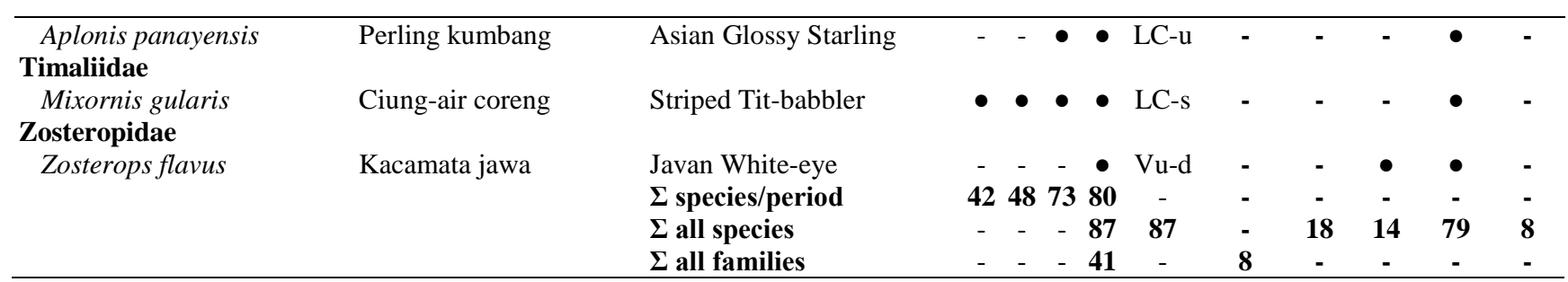

Note: Observation period: P1 (8 - 15 April 2015), P2 (29 July - 4 August 2015), P3 (5 - 12 November 2015), and P4 (28 February - 8 March 2016). Waterbirds: Fam. = family; Sp. = species; $L C=$ least concern; $N T=$ near threaten; $V u=$ vulnerable. $i=i n c r e a s i n g ; ~ d=$ decreasing; $\mathrm{s}=$ stable; $\mathrm{u}=$ unknown. Occupancy: Res. = resident; Mig. = migrant. Prot. = protected by the Regulation of the Minister of Environment and Forestry, the Republic of Indonesia No. P.92/MENLHK/SETJEN/KUM.1/8/2018, 30 August 2018

Waterbirds can also be recorded in small ponds scattered in the complex. The size of the pond varies from around $20 \mathrm{~m}^{2}$ to $500 \mathrm{~m}^{2}$ with a maximum depth of around 3 $\mathrm{m}$. Some ponds are usually visited by fish anglers. Waterbirds commonly recorded in ponds like this include Striated Heron and Little Egret. This bird can be found in the pond if there are no humans (such as anglers, lawn mowers) near the pond. If there is, the position of humans is relatively far from birds. The activity is not excessive or does not disturb the birds.

The number of birds in the Tarjun Factory Complex should be compared to that in the same complex according to the observation period to analyze the development of birds in the complex. However, this cannot be done because there were no previous reports. However, when compared to data from other ITP factory complexes, namely Citeurep and Palimanan and without differentiating their habitat types, the number of birds in Tarjun is slightly larger.

The number of species that are classified as numerous and varied in the Tarjun Factory Complex may be due to three factors. First, the ITP complex has a diversity of landscapes (waters, grasslands, vegetation areas) and also plant diversity growing on it. The quality of landscapes, as well as, plants gradually increases in accordance with age and the positive treatments given to it. In the beginning, the landscape can be categorized as artificial and some plants that grow and develop on it are intentionally planted. Habitat conditions with various species of life or life interactions between physical components make a safe and comfortable habitat for birds. Different habitat from birds caused the feed resources (quantity and quality), competition between fauna in the resources (space and time).

The complex was built by another company before according to ITP (2018a), was acquired by ITP on December 29, 2000. Indeed there is no data related to flora fauna species in the landscape published to the public. However some plants that were visually identified intentionally planted were forestry plants, such as Acacia mangium, A. auriculiformis, mahoni (Swietenia macrophylla), sengon (Paraserianthes falcataria), trembesi (Samanea saman), horticultural crops, such as patai (Parkia speciosa), mango (Mangifera spp.), watery roseapple (Syzygium aqueum), apple guava (Psidium guajava), and banana (Musa spp.), and decorative plants, such as peacock flower (Caesalpinia pulcherrima), asoka (Ixora spp.), and Hippobroma longiflora. In addition, some plants grow spontaneously, such as bamboo, various species of mahang (Macaranga spp.), karamunting (Rhodomyrtus tomentosa), ketapang (Terminalia catappa), alaban (Vitex pubescens), kirinyuh (Chromolaena odorata), tembelekan (Lantana camara), Poaceae, and Cyperaceae.

Changes or differences in habitats affect animals; in this discussion birds. The landscape gradients affect the diversity and distribution of avifauna (Altaf et al. 2018). A gradient from arable fields to forest affects farmland and forest avifauna species (Kosickia and Chylarecki 2012). The diversity of habitat types which are reflected by physical conditions (watery or not, there are buildings or not, there are human activities or not) and biological conditions (plant species that form habitats and their vegetation strata) triggers bird species diversity (Soendjoto et al. 2014). The habitat changes from natural forest to secondary forest, land with agroforestry systems, and land with annual cultures gradually alter the composition of bird species (Waltert et al. 2004). The number of bird species in the reclaimed and revegetated ex-mining sites in South Kalimantan Province was increased as long as the vegetation in the site was preserved (Soendjoto et al. 2018). The old age stands of large-scale oil palm plantations have more diverse birds than the young one (Erniwati et al. 2016). The diversity or abundance of bird communities on oil palm plantations is worse than in natural forests (Teuscher et al. 2015); likewise in rice fields or roads when compared to agroforestry (Tanalgo et al. 2015). Microclimate, as well as both comfortable and safe conditions on vegetation and vegetated land, are needed animals, including birds need for resting, playing, hiding from predators, voicing, and even breeding (Soendjoto et al. 2015a).

Second, the conditions inside the ITP complex are balanced by the conditions of the surrounding environment which in this case also consist of landscape diversity and plant diversity. The latter landscape consists of the Terusan River, which is more than $30 \mathrm{~m}$ wide and more than $5 \mathrm{~m}$ deep, mangrove forests, lowland secondary forests and oil palm plantations. The plants are diverse. Bakau (Rhizophora apiculata, R. mucronata), rambai/mangrove apple (Sonneratia caseolaris), rambai bogam (S. alba), and langgadai/large-leafed mangrove (Bruguiera gymnorrhiza) are some main plants of the mangrove ecosystem. Alaban, 
ketapang, piai (Acrostichum aureum), bintaro (Cerbera manghas), and rattan spontaneously develop in lowland secondary forests. Oil palm is the main crop in the largescale plantations. Woody plants are not allowed to live on oil palm plantations. Plants commonly found in oil palm plantations are wild plants, such as ferns and grasses. The others are plants that are intentionally planted to divert pest and disease vectors to attack oil palm. The latter plant is Turnera subulata, a flowering plant of the Turneraceae family.

Landscape and plants outside the ITP complex are very useful for birds. This area can be used by birds at least to save themselves. On the other side, although the movement of birds or animals from one place or another does not recognize administrative boundaries, this limit can be used as an imaginary line that distinguishes habitat types or human treatments in habitat (anthropogenic factors). When the birds inside the complex are disturbed, they fly or move to adjacent landscapes outside the complex. Factors that may play an important role in the similarity of bird communities are the short distance between two adjacent forest types, the abundance of insect food sources, and the succession stages similar to forest types (Kaban et al. 2017). Hunting can make a bird behavior change, in the form of increased chances of flying, more time wasted on alert, and less rest or bird move from hunting areas to areas where there is no hunting (Casas et al. 2009).

Third, the imposition of a hunting ban on ITP complex facilitates birds to be safe and comfortable from interference. Hunting ban boards that also contain sanctions for violations of these regulations are installed in strategic places (such as public transport roads and lakeside) that are easily seen by employees or the public entering the complex. The ban is obeyed by them. In turn, it has a positive impact. Under certain conditions, birds can adapt physiologically and behaviorally (Sokos et al. 2013). Birds are accustomed to these conditions so that population and composition or in general bird diversity increases gradually.

The causes of the reduction in bird populations are not only deforestation (Symes et al. 2018), but also bird hunting for food, used as pets, traded, or other purposes (Fernandes-Ferreira et al. 2012, Iskandar et al. 2016). Hunting can change animal community composition and may affect a large number of ecological interactions (Barbosa et al. 2014) and jeopardizes the survival of birds, especially waterbirds (Wang et al. 2018) and migratory bird species (Arizaga and Laso 2015). Although not a few researchers argue that hunting endangers the survival of bird species, some researchers who argue otherwise. Hunting has no significant effect on bird abundance (Sulaiman et al. 2014).

In addition to the total number of bird species found (including waterbirds), bird protection status, and bird occupancy status, there are results of research in the ITP factory complex that needs attention. The number of bird species found in periods 1 and 2 is smaller than in periods 3 and 4.

The main objective of this study is not to distinguish birds in the dry and rainy seasons. Thus, a mere coincidence, if there are differences in the number of bird species in periods 1 and 2 compared to periods 3 and 4 . In the case of climate in Indonesia, April - October is called the dry season. Conversely, October - April is the rainy season. In the dry season, grass generally dries. The leaves of some trees even fall out. This also happened in the Tarjun Factory Complex. Exceptions only occur in the grass on the golf course. The grass here remains green because it is treated (watered and cut) every day.

In the dry season, plants as food sources for birds in the factory complex are not available or not enough to meet the nutritional needs of birds. Conditions like this force the birds to be more active in spreading outside the complex which has sufficient food sources. Spreading to wider or farther areas can be done by birds in a short time because these animals can fly to move from unsafe and uncomfortable places to other places that are safer and more comfortable. Not surprisingly, if later within a relatively short duration of observation, i.e., 2 hours in the morning and 1.5 hours in the afternoon the number of bird species observed were 42 and 48 respectively.

The opposite happened in the rainy season. Plants are fresh and green. Most plants even produce fruit and stimulate insects to come, so that the plant environment provides enough food for birds. Birds also do not need to spread to find food to another place farther away. In this condition, 73 species of birds in period 3 and 80 species in period 4 were recorded. This size includes migratory birds with relatively few species (only 8 species or even 7 species, if Java Sparrow is removed from the category of migratory birds).

\section{ACKNOWLEDGEMENTS}

We would like to thank the ITP Manager at Tarjun, South Kalimantan who financed and facilitated this research. Further our sincere gratitude also goes to Wisnu Adi Patria for extensive support given during field surveys.

\section{REFERENCES}

Altaf M, Javid A, Khan AM, Khan MSH, Umair M, Ali Z. 2018. Anthropogenic impact on the distribution of the birds in the tropical thorn forest, Punjab, Pakistan. Journal of Asia-Pacific Biodiversity 11(1): 229-236. DOI 10.1016/j.japb.2018.03.001.

Arizaga J, Laso M. 2015. A quantification of illegal hunting of birds in Gipuzkoa (North of Spain). Eur J Wildl Res 61:795-799. DOI 10.1007/s10344-015-0940-6.

Barbosa EDO, da Silva MGB, de Medeiros RO, Chaves MF. 2014. Hunting activities impact avifauna in rural areas of the Jaçanã municipality, Rio Grande do Norte State, Brazil. Biotemas 27(3): 175-190.

Casas F, Mougeot F, Vinuela J, Bretagnolle V. 2009. Effects of hunting on the behaviour and spatial distribution of farmland birds: importance of hunting-free refuges in agricultural areas. Anim Conserv 2009: 1-9.

Erniwati, Zuhud EAM, Santosa Y, Anas I. 2016. The value of secondary forest patches for bird conservation in palm oil landscapes of Riau,

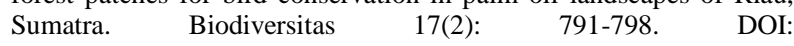
10.13057/biodiv/d170256.

Fernandes-Ferreira H, Mendonc a SV, Albano C, Ferreira FS, Alves RRN. 2011. Hunting, use and conservation of birds in Northeast Brazil. Biodivers Conserv 21(1): 221-244. DOI 10.1007/s10531-011-0179-9. 
MacKinnon J, Phillipps K, Balen B. 2010. Birds in Sumatra, Java, Bali, and Kalimantan (Including Sabah, Sarawak and Brunei Darussalam) Burung Indonesia, Bogor, Indonesia. [Indonesian].

Heidelberg Cement $\quad$ Group. $2018 . \quad$ Indonesia. https://www.heidelbergcement.com/en/indonesia.

Iskandar J, Iskandar BS, Partasasmita R. 2016. The local knowledge of the rural people on species, role and hunting of birds: Case study in Karangwangi Village, West Java, Indonesia. Biodiversitas 17(2): 435 446. DOI 10.13057/biodiv/d170206.

ITP. 2015. Sustainability Report 2014. Improving Efficiency, Achieving Sustainability.

http://www.indocement.co.id/v5/IndocementContent/Attachment/Rep ort/Sustainability\%20Report/03\%20SR\%20INDOCEMENT\%202014 .pdf.

ITP. 2016. Sustainability Report 2015. Ensuring Sustainability for the Next Decades. http://www.indocement.co.id/v5/IndocementContent/Presentation/201 $6 / 160418 \% 20-$

\%20AR\%20SR/Indocement\%20Sustainability\%20Report\%202015.p df.

ITP. 2017. Sustainability Report 2016. Keeping a Blue Sky: Being Different to Shape the Future http://www.indocement.co.id/v5/IndocementContent/Presentation/201 7/ARSR2016/SR\%20Indocement\%202016_lowres.pdf.

ITP. 2018a. Indocement in http://www.indocement.co.id/v5/en/company/indocement-in-brief/

ITP. 2018b. Indocement Opens New Cement Terminal \& Aims for LowEnd Market. Indonesia-Investments. https://www.indonesiainvestments.com/news/todays-headlines/indocement-opens-newcement-terminal-aims-for-low-end-market/item 8676 ?

ITP. 2018c. PT Indocement Tunggal Prakarsa Tbk. Q1 2018 Results. http://www.indocement.co.id/v5/IndocementContent/Press\%20Releas e/2018/Indocement\%20Q1\%202018\%20Results_20180518.pdf.

ITP. 2018d. Sustainability Report 2017. Agility for Sustainability. http://www.indocement.co.id/v5/IndocementContent/GCG/2018/SR\% 20Indocement $\% 202017 . p d f$.

IUCN. 2018. The IUCN Red List of Threatened Species. Version 2018-1. <www.iucnredlist.org>. Downloaded on 14 November 2018.

Kaban A, Mardiastuti A, Mulyani YA. 2017. Response of bird community to various plantation forests in Gunung Walat, West Java, Indonesia. Hayati J Biosci 24: 72-78.

Kosickia JZ, Chylarecki P. 2012. Effect of climate, topography and habitat on species-richness of breeding birds in Poland. Basic Appl Ecol 13: 475-483. DOI 10.1016/j.baae.2012.07.007.

Smythies BE. 1981. The Birds of Borneo. The Sabah Society \& The Malayan Nature Society, Kuala Lumpur.

Soendjoto MA, Riefani MK, Mahrudin, Zen M. 2014. Dynamics of avifauna species in the area of PT Arutmin Indonesia - North Pulau Laut Coal Terminal, Kotabaru, South Kalimantan. In: Karyanto P et al. (Eds). Proceedings of National Conference XI on Biology Education. Sebelas Maret University, Surakarta, 7 June 2014. Pp. 512-520. [Indonesian].

Soendjoto MA, Riefani MK, Triwibowo D, Anshari MN, Metasari D. 2015a. Wild animals in the reclamation area of PT Adaro Indonesia, South Kalimantan revegetated less than two years. In: Sajidan et al. (Eds.). Proceeding of National Conference on Conservation and Utilization of Nature Resources, Sebelas Maret University, Surakarta, Indonesia, 13 Januari 2015. pp. 192-198. [Indonesian].

Soendjoto MA, Riefani MK, Triwibowo D, Metasari D. 2018. Birds observed during the monitoring period of 2013-2017 in the revegetation area of ex-coal mining sites in South Kalimantan, Indonesia. Biodiversitas 19(1): 323-329. DOI 10.13057/biodiv/d190144.

Soendjoto MA, Riefani MK, Triwibowo D, Wahyudi F. 2015b. Avifauna in the Reclamation Area of PT Adaro Indonesia. Banjarbaru: Universitas Lambung Mangkurat Press. [Indonesian].

Sokos CK, Birtsas PK, Connelly JW, Papaspyropoulos KG. 2013. Hunting of migratory birds: disturbance intolerant or harvest tolerant? Wildl Biol 19: 113-125. DOI 10.2981/12-032.

Strange M. 2002. A Photographic Guide to the Birds of Southeast Asia, Including the Philippines \& Borneo. Periplus Editions (HK) Ltd., Hong Kong.

Sulaiman IM, Apeverga PT, Ringim AS, Ibrahim KM. 2014. Effects of anthropogenic activities on bird diversity and abundance at the Hadejia - Nguru Wetlands, Nigeria. Stand Sci Res Essays 2(10): 546551.

Symes WS, Edwards DP, Miettinen J, Rheindt FE, Carrasco LR. 2018. Combined impacts of deforestation and wildlife trade on tropical biodiversity are severely underestimated. Nat Communications 9(4052).

Tanalgo KC, Pineda JAF, Agravante ME, Amerol ZM. 2015. Bird diversity and structure in different land-use types in lowland SouthCentral Mindanao, Philippines. Trop Life Sci Res. 26(2): 85-103.

Teuscher M, Vorlaufer M, Wollni M, Brose U, Mulyani Y, Clough Y. 2015. Trade-offs between bird diversity and abundance, yields and revenue in smallholder oil palm plantations in Sumatra, Indonesia. Biol Conserv 186: 306-318.

Waltert M, Mardiastuti A, Mühlenberg M. 2004. The effect of land use on bird species richness in Sulawesi, Indonesia. Conserv Biol 15:13391346. DOI 10.1111/j.1523-1739.2004.00127.x.

Wang X, Kuang F, Tan K, Ma Z. 2018. Population trends, threats, and conservation recommendations for waterbirds in China. Avian Res 9:14. DOI 10.1186/s40657-018-0106-9.

Wetlands International. 2012. Waterbird Population Estimates, Fifth Edition. Summary Report. Wetlands International, Wageningen, The Netherlands. 